Teknokultura. Revista de Cultura Digital y Movimientos Sociales

ISSNe: $1549-2230$

http://dx.doi.org/10.5209/TEKN.69366

\title{
Entre emprendedores culturales e inversores inmobiliarios: crowdfunding en España 2015-2019
}

\author{
Elena Gil Moreno ${ }^{1}$
}

Recibido: 10 de mayo de 2020 / Aceptado: 14 de julio de 2020 Open peer reviews

Resumen. Este artículo aborda algunas de las características del sector del crowdfunding en España, haciendo una comparativa entre datos primarios de 2015 y 2019. A través de este estudio observamos cómo el crowdfunding ha ido dejando parte de su cultura colaborativa para convertirse en un sector financiero fuertemente influido por las empresas inmobiliarias. Al observar esta nueva cara del sector es lógico cuestionarse si el crowdfunding es realmente un fenómeno colaborativo. Los resultados han sido obtenidos a raíz de una investigación iniciada en 2014, que se encuentra en permanente actualización.

Palabras clave: economía colaborativa; industrias culturales; internet; prosumo.

\section{[en] Between DIY artists and real estate investors: crowdfunding in Spain between 2015 and 2019}

Abstract. The article addresses some of the characteristics of the Spanish crowdfunding sector by comparing primary research data from 2015 and 2019. The results show that crowdfunding has gradually left behind much of its collaborative culture to become a financial sector strongly influenced by real estate companies. This new dimension of the sector calls into question the positioning of crowdfunding as a collaborative phenomenon. The data used in the study was drawn from a research project that began in 2014 , which is constantly updated.

Keywords: cultural industries; Internet; prosumption; sharing economy.

Sumario. 1. Introducción. 2. Estrategia metodológica. 3. Hacia una definición del crowdfunding. 4.2015 vs 2019 ¿Dos fenómenos diferentes o el mismo? 5. El trabajo desde las narrativas liberadoras o desde la autoexplotación. 6. Conclusiones. 7. Referencias.

Cómo citar: Gil Moreno, E. (2021). Entre emprendedores culturales e inversores inmobiliarios: crowdfunding en España 2015-2019. Teknokultura. Revista de Cultura Digital y Movimientos Sociales, 18(1), 13-22.

\section{Introducción}

Las economías colaborativas emergieron tras el estallido de la crisis financiera. El interés científico por lo "colaborativo" surge en torno a 2009 coincidiendo con la aparición de importantes iniciativas tales como Kickstarter, Airbnb o Uber. La ausencia total de referencias en la literatura por aquel entonces, así como la pertinencia de los temas tratados, por ser asuntos de actualidad que están modificando las formas de trabajo y de consumo, provocaron una creciente red de investigaciones que han intentado describir y analizar lo colaborativo desde perspectivas economicistas, sociológicas y de la comunicación. Algunos autores han problematizado lo colaborativo desde el análisis de las dinámicas de prosumo (O’Neil y Frayssé, 2015; Ritzer y Jurgenson, 2010) y la reconceptualización de la "macdonalización" de nuestras sociedades. Otros han comprendido los patrones de comportamiento de los prosumidores de lo colaborativo, reinterpretando las teorías de los commons y abriéndose paso hacia otras perspectivas que atienden a las formas del commoning (Bollier y Helfrich, 2015). La literatura ha traído consigo debates sobre las bondades de las sociedades colaborativas a través del análisis de sus "narrativas liberadoras" (Manzini, 2019) y la reproducción de dinámicas de opresión desde "el entusiasmo" (Denegri-Knott y Zwick, 2012; Zafra, 2017).

A pesar del interés de este tipo de análisis se siguen echando en falta estudios empíricos concretos. No es posible comparar el crowdfunding, en el que existen numerosas plataformas, con el transporte colaborativo donde encontramos "empresas unicornio" que dominan el mercado, como en el caso de Uber o Cabify. Aileen Lee, fundadora del fondo de inversión Cowboy Ven- 
tures, acuñó este término para referirse a las empresas tecnológicas que alcanzan un valor de 1000 millones de dólares sin tener presencia en Bolsa, por ejemplo: Facebook (Lee, 2013). Si nos centramos en el crowdfunding, podremos observar que no existen "empresas unicornio" en nuestro país. Por el contrario, encontramos numerosas PYMES, así como asociaciones, fundaciones, organismos públicos, cooperativas, startups y en los últimos años también empresas con más poder de facturación procedentes del sector inmobiliario. El análisis del crowdfunding en España tiene que pasar irremediablemente por un estudio descriptivo lo más riguroso posible que permita entender las características del sector.

En este texto vamos a trazar una línea temporal entre 2015 y 2019, siendo este el periodo del que hemos conseguido tener una base de datos actualizada sobre el sector en España. Observaremos que se han producido enormes cambios en tan solo cuatro años y trataremos de describir sus características. De este modo, en el futuro podremos conceptualizar mejor los análisis de lo colaborativo asociado al crowdfunding y saber si tiene sentido seguir hablando de economía colaborativa o no, si existen peligros relacionados con las prácticas de consumo en el crowdfunding, si ha sido un fenómeno temporal hoy en decadencia o, por el contrario, sigue en expansión, entre otras cuestiones.

\section{Estrategia metodológica}

Un problema es la incapacidad que tienen los investigadores de lo colaborativo para acceder a datos fidedignos con los que trabajar sus estudios. No existen censos estatales que digan cuántas plataformas de bancos del tiempo hay en nuestros países, cuáles son las empresas de coworking, la cantidad de huertos compartidos, o las iniciativas para compartir bicicleta en distintas ciudades. A lo sumo, podemos encontrar directorios elaborados por entusiastas de lo colaborativo, o generalmente por consultores que se han dedicado a indagar en Internet y han cruzado sus tentativas de investigación con sus conocimientos personales para ofrecer a través de sus blogs una estimación de las plataformas existentes en nuestros países, por ejemplo el blog "Consumo Colaborativo" (2017). Esta escasez de datos fiables e independientes puede haber influido en las investigaciones sobre lo colaborativo, normalmente centradas en estudios de caso de "empresas unicornio" y sus efectos sobre el prosumo. Incluso podemos encontrar un creciente interés social por la regulación y mejora de las prácticas de algunas de estas empresas, como demuestra la sentencia de 2019 que dictamina que los riders de Deliveroo son falsos autónomos (Sabadell, 2019).

Los datos presentados en este texto forman parte de una investigación iniciada en 2014 y en la que seguimos trabajando hasta la fecha. Durante los dos primeros años se realizó un análisis netnográfico de blogs, webs y perfiles de redes sociales relacionado con el crowdfunding en España. En esta primera fase centramos nuestros esfuerzos en la elaboración de un directorio actualizado de este tipo de plataformas. En 2017 el directorio contaba con 135 plataformas activas e inactivas, hoy lo hace con 159 (E. Gil, 2019, pp. 176-177). Para su elaboración se tuvieron en cuenta otros listados como el de la consultora Crowdacy y la Asociación Española de Crowdfunding. Se utilizó la búsqueda directa de plataformas a través de Google y de las opciones de búsqueda de Twitter. Durante su elaboración recurrimos a una campaña de crowdfunding para realizar observación participante, lo que incluyó la creación de una cuenta personal de Twitter que sirvió como presentación de la investigación al sector. El proyecto no puede ser localizado por el cierre de la web de la plataforma, así como del propio periódico. Sin embargo, dio como resultado un artículo titulado "El crowdfunding en España. ¿Qué modelo estamos creando?" (Gil Moreno, 2014, 8 de abril) ${ }^{2}$. La campaña fue lanzada en la plataforma "Información Sensible", una iniciativa periodística, y dio lugar a la escritura de un artículo de prensa publicado en el periódico digital. Tras la publicación de este artículo se creó un blog en el que permanecía fijada una entrada con el directorio de plataformas actualizado.

Twitter resultó ser una buena herramienta de búsqueda de información a través de la cual empresas que querían aparecer en el listado utilizaban la opción del follow o escribían solicitando su presencia en el mismo. Aunque el directorio no es ni puede ser un censo exacto, es el listado más completo hasta la fecha con el que contamos para estudiar el sector. Se procedió a crear una base de datos que contuviera información relevante sobre las plataformas, que fue recogida a través de fuentes primarias, creando variables $(n=24)$ con información sobre la ciudad y comunidad autónoma donde fueron creadas, el año en que se fundaron, o si existían apoyos externos en forma de partenariado o algún tipo de colaboración. Para obtener estos datos se navegó en sus webs recopilando la información, que solía aparecer en los apartados de preguntas frecuentes (FAQs) así como en las secciones del aviso legal, quiénes somos, entre otros. Una buena parte de los datos buscados no pudieron ser obtenidos por observación directa, por ello se lanzó un cuestionario online entre octubre de 2014 y enero de 2015. Fue enviado a través de e-mail a todas las plataformas activas del directorio, un total de 82 . Se realizaron tres recordatorios publicitados tanto vía e-mail como a través de Twitter. La tasa de respuesta fue del $64 \%$.

Además, se inició un proceso de investigación cualitativa en el que se realizaron dos grupos focales con plataformas de donación y de préstamo en la ciudad de Barcelona durante el año 2015. También realizamos dos entrevistas en profundidad a personas clave del sector.

\section{Hacia una definición del crowdfunding}

El crowdfunding es un fenómeno online que irrumpió en nuestras sociedades tras el estallido de la crisis financiera de finales de la década pasada. Resaltamos que es un fenómeno online, ya que son comunes las declaraciones que afirman que el crowdfunding siempre ha existido como práctica social en espacios analógicos, poniendo

https://crowdthescience.wordpress.com/ 
como ejemplos la financiación de la Estatua de la Libertad o a Lola Flores con su famoso "una peseta de cada español" para pagar su multa con Hacienda (Rey, 2016). Aunque podemos entender estos y otros ejemplos como antecedentes, el hecho de que exista una plataforma digital con una estructura web que permite hacer unas cosas y otras no, importa. Si seguimos la perspectiva del actorred, mecenas, promotores de proyectos y plataformas van conformando a través de la performatividad el cómo del fenómeno. ¿Se trata de romper con las sociedades tradicionales? ¿Crean proyectos más democráticos? ¿Forman parte de identidades digitales? Sin embargo, al mismo tiempo, los usuarios no tienen total libertad para definir qué "usos" van a dar a esas tecnologías (Lasén, 2006). Al contrario, están condicionados por el diseño de estas plataformas web. ¿Permiten pagar desde fuera del país? ¿Pueden hacer comentarios debajo de los proyectos? Crowdfunding en esta investigación implica necesariamente la existencia de plataformas digitales que hacen de intermediarias entre mecenas y promotores de proyectos.

El crowdfunding, además, se aleja del concepto de fundraising, que hace referencia al modelo de captación de fondos de las ONGDs. A diferencia de este, lo entendemos como una forma de consumo colaborativo, ya que refleja muchos de los valores de la cultura colaborativa, como la existencia de "un intercambio monetario, material o no material" (Belk, 2014, p. 1597) o la conexión de "individuos y comunidades de manera descentralizada en oposición a las instituciones económicas asentadas, transformando como producimos, consumimos, nos financiamos y aprendemos" (Botsman y Rogers, 2010, p. 13). Aunque no está en principio sustentado en otras investigaciones académicas, sí que encontramos evidencias que per- miten definir quiénes son los pioneros del crowdfunding. Las primeras plataformas suelen estar relacionadas con el mundo de las artes, lo cultural, lo creativo dando lugar a lo que en esta investigación hemos llamado el modelo "creativo-cultural", representado como "ideal-tipo" por la plataforma Kickstarter. Se trata de modelos de negocio en los que "emprendedores culturales" lanzan proyectos para su financiación. Los entenderemos como aquellos artistas alejados de la industria cultural y también de las industrias creativas (Rowan, 2010) es decir, la categoría más baja en una escala de precariedad dentro del mundo de las artes. Artistas que tienen ideas, pero escasos recursos y redes de contactos de bajo impacto.

Los pioneros del crowdfunding son, por tanto, los trabajadores más pobres de un sector ya de por sí precarizado. Esto provocó una percepción social del crowdfunding ligada a los discos y las películas. Sin embargo, ha evolucionado incorporando a sectores diversos como el mundo editorial, la investigación y desarrollo $(\mathrm{I}+\mathrm{D}+\mathrm{i})$, el periodismo, así como la expansión del crowdfunding financiero. Aunque ya existían plataformas como ArtistShare (2000) o SellABand (2006), no es hasta 2009 que observamos una explosión del fenómeno a nivel internacional con el lanzamiento de IndieGogo (2008) y Kickstarter (2009). Es precisamente este año en el que en España se funda Lánzanos, nuestra primera plataforma de crowdfunding. El fenómeno se expande hacia América del Norte, Europa y Asia. De acuerdo con los datos de la consultora Massolution, en 2014 casi el 80\% del crowdfunding se concentraba en Europa y América del Norte (2015). Estábamos ante un fenómeno claramente influido por el impacto de la crisis financiera global y en principio, medianamente occidental (véase Figura 1).

Figura 1. Número de plataformas de crowdfunding en Europa en el año 2014.

Fuente: Wardrop et al. (2015, p. 14)

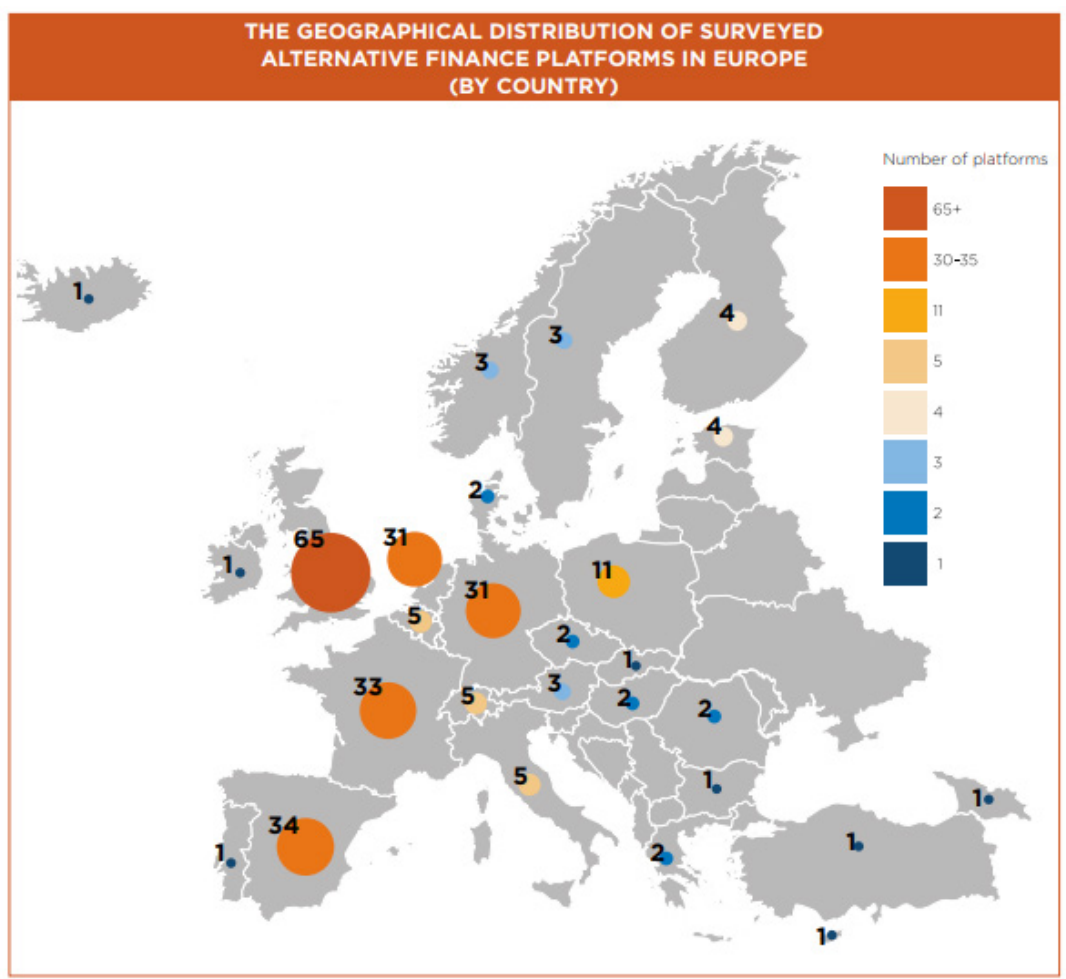


El consenso en la literatura académica y en el propio sector afirma que existen al menos cuatro modelos de financiación. El modelo de recompensas, ligado a los "emprendedores culturales" y expandido a otros perfiles de promotores de proyectos, en el que se ofrecen intercambios materiales e inmateriales a los mecenas. En este modelo se sitúan plataformas como Verkami, Lánzanos o Goteo. También el modelo de donación, conectado habitualmente con proyectos de carácter social y solidario, así como a fundaciones y ONGD's que operan como intermediarias entre los promotores de proyectos y los mecenas, tales como Mi Grano de Arena o Teaming. Por otro lado, encontramos lo que la Comisión Europea (2013) ha denominado "crowdfunding financiero". Se trata de los modelos que nuestro marco regulatorio exige estén supervisados por la Comisión Nacional del Mercado de Valores (CNMV) (Ley 5/2015, de 27 de abril, de fomento de la financiación empresarial, 2015). Estos modelos se dividen entre el crowdfunding de préstamo $\mathrm{y}$ el de inversión. El primero promueve la financiación de créditos peer-to-peer ( $\mathrm{P} 2 \mathrm{P})$ donde la recompensa es monetaria. LoanBook o Finanzarel son buenos ejemplos de este modelo. El segundo ofrece el retorno financiero en forma de acciones ganadas por la puesta en marcha de proyectos, con plataformas como The CrowdAngel o Capital Cell, pero también con la incorporación desde 2016 de plataformas inmobiliarias como Housers o Privalore.

Según el último informe anual realizado por la consultora Universo Crowdfunding y la Universidad Complutense de Madrid, el crowdfunding recaudó en nuestro país 418.178.546€ desde 2013 (González y Ramos, 2018) (Gráfico 1). Una cifra considerablemente elevada que ha influido seguramente en el interés de la Comisión Europea por crear un marco regulatorio del sector $(\mathrm{Eu}-$ ropean Commission, 2013).

Gráfico 1. Evolución de la recaudación monetaria acumulada según modelos de crowdfunding (España, 2018).

Fuente: González y Ramos (2018, p. 10)

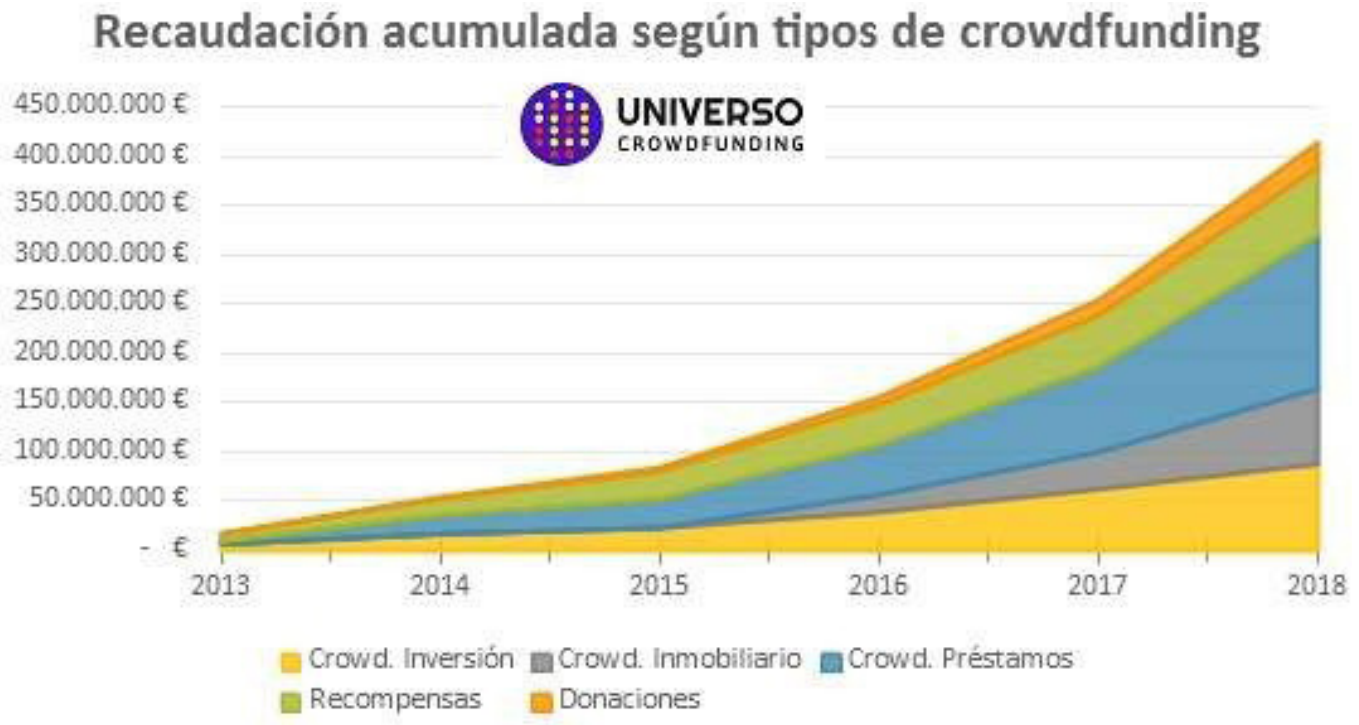

Si observamos la cantidad de dinero recaudado según modelos, podremos comprobar que la donación y las recompensas han quedado relegadas a un segundo plano en contraposición a los modelos financieros representados por el crowdfunding de préstamo y de inversión, entre el que se encuentra el inmobiliario. Estos datos se encuentran en la línea de las conclusiones generales de este estudio, donde se defenderá que la irrupción del crowdfunding inmobiliario ha modificado notablemente la cara pública o el perfil de este sector.

\section{2015 vs. 2019 ¿Dos fenómenos diferentes?}

Durante los primeros años, alrededor de 2009, no estaba claro si el crowdfunding constituía un fenó- meno que pudiera ser sostenido en el tiempo, o si por el contrario nos encontrábamos ante un boom del sector que iba a ir disipándose poco a poco. Tras contrastar nuestros datos, podemos afirmar que entre 2011 y 2014 se crearon en España una fuerte cantidad de plataformas, dándole al sector una imagen de éxito por su carácter expansivo (Gráficos 2 y 3). En el intervalo de estos años se crearon el $77.30 \%$ del total de las plataformas, pero debemos resaltar que el $51.06 \%$ de ellas ya no están en activo. Más de la mitad de las plataformas creadas desde 2007 ya no existen. Algunas, como EcoCrowdfunding, se han reconvertido en consultoras siguiendo dentro del sector, pero sin ejercer como intermediarias entre mecenas y promotores. 
Gráficos 2 y 3. Evolución temporal del crowdfunding en España. Fuente: elaboración propia.

\section{Evolución del crowdfunding en España}

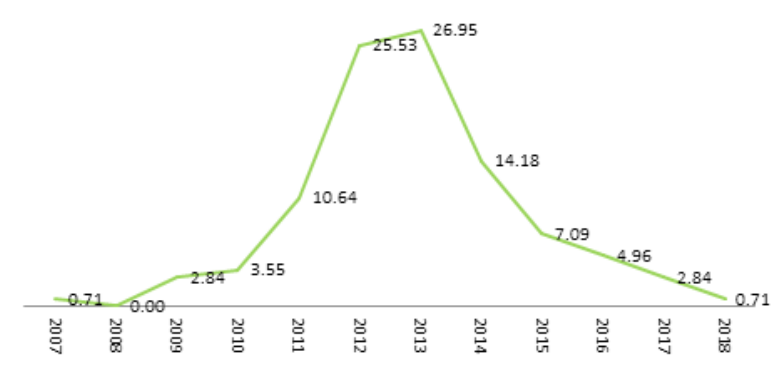

Si nos centramos en los modelos definidos con anterioridad, observamos que el modelo de recompensas y
Evolución del crowdfunding en España

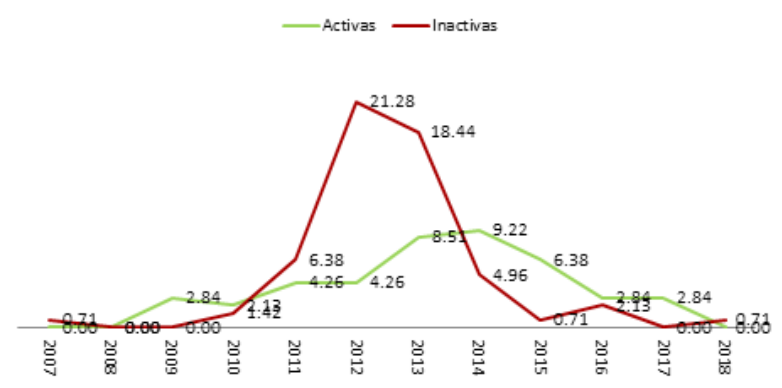

el de donación descienden mientras el de inversión asciende (Gráfico 4).

Gráfico 4. Evolución temporal del crowdfunding según modelos en España.

Fuente: elaboración propia

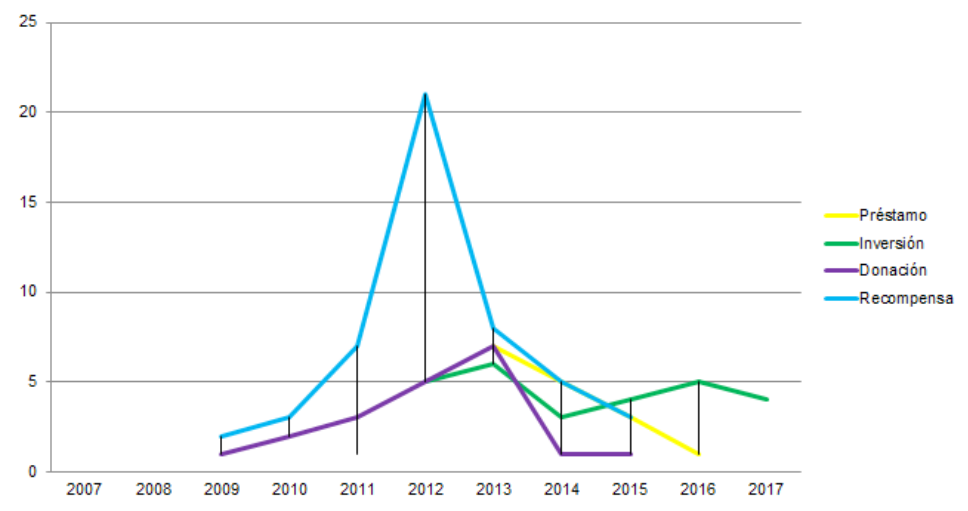

No obstante, siguiendo los datos ofrecidos por Universo Crowdfunding y la Universidad Complutense, 2016 sería un año clave para la expansión de un sector que recauda cada vez más dinero (González y Ramos, 2018). El perfil de mecenas de los modelos financieros y el resto difieren en forma y capacidad adquisitiva. Así lo reflejaba Carlos Otto (2017) en un artículo de El Confidencial en el que desgranaba el perfil de dos inversores de la plataforma The Crowd Angel: Ángel Cano, consejero delegado del BBVA y despedido por su banco y Manuel Galatas, ex director general del BBVA en Asia y Turquía. El crowdfunding de inversión aparece como una nueva salida para los flujos financieros por parte de perfiles con altas capacidades adquisitivas en lugar de una forma de consumo colaborativo.

La conceptualización de qué es economía colaborativa y qué no, es compleja. Los medios y el modo en que se autodefinen las propias plataformas deciden en buena medida quién forma parte y quién no del supuesto consumo colaborativo (Schor, 2014). De este modo, las plataformas de donación se han estado definiendo como parte de iniciativas de consumo colaborativo, a pesar de que su discurso y prácticas se parecen al fundraising tradicional de las ONGD's pero mediado por Internet. Las plataformas de préstamo han reproducido un discurso asociado a conceptos como la desbancarización, la desintermediación financiera o defendido su presencia en el sector como sujetos activos que facilitan la trans- versalidad de los créditos financieros a personas con menor capacidad adquisitiva. Finanzarel publicaba en su cuenta de Twitter el 1 de octubre de 2015 "Los jóvenes piden un cambio en la \#banca, \#financiación alternativa es la opción preferida". El 25 del mismo mes se hacían eco de un artículo de El Confidencial y escribían "En@ elconfidencial: ¿Miedo de las entidades financieras a la transparencia?" o al día siguiente publicaban en El Economista "La \#pyme española acude a las plataformas \#fintech, la nueva tendencia de \#financiación".

La defensa de plataformas como Finanzarel de acercar el crédito hacia las PYMES, que pueden generar puestos de trabajo, es clara y encaja bien en un discurso post-crisis y colaborativo de crear alternativas sociales donde se redistribuyan las tradicionales brechas económicas asociadas al componente de clase social. Son plataformas que se presentan como sujetos políticos del cambio hacia sociedades más colaborativas, horizontales y transparentes.

Lo mismo sucede con las plataformas inmobiliarias. Housers en su página web, se define como "la primera plataforma de ahorro e inversión que ha revolucionado las reglas del juego haciendo posible la democratización de la inversión en activos reales". The Urban Investors por su parte, afirma también en su web "Inversión inmobiliaria. Probablemente te suene a grandes empresas, a apellidos poderosos. A rentabilidades tan altas como los edificios que se construyen $\mathrm{y}$, al mismo tiempo, tan 
alejadas de los ciudadanos... Pero ¿Y si utilizamos la tecnología para democratizarla? ¿Y además lo hacemos en las mejores ciudades?". El discurso se presenta contradictorio con la realidad. Tal y como afirma Hernández (2017, p. 24), estamos ante una reconversión del sector inmobiliario alejada del ethos de lo colaborativo:

En España, existen varias plataformas (Privalore, BricksyPeople, Peerbrick y FT Inversión) que publicitan crowdfunding inmobiliario articulado jurídicamente mediante contratos de cuentas en participación. Todas estas plataformas han sido creadas y están bajo el control de empresas promotoras o constructoras que solicitan financiación para proyectos propios. Estas empresas promotoras o constructoras ofrecen al público, a través de una plataforma online de su propie- dad o bajo su control, la posibilidad de invertir en sus propios proyectos de construcción o rehabilitación, articulando esa inversión mediante múltiples contratos de cuentas en participación con los inversores. Por lo tanto, la plataforma no actúa como intermediario entre demandantes y oferentes de capital, sino que es un medio instrumental a través del cual un promotor solicita públicamente financiación para sus propios proyectos inmobiliarios (2017, p. 24).

Precisamente, el hecho de que no sean, tal y como la autora afirma, plataformas intermediarias, aleja totalmente a este sector del concepto de economías colaborativas, dado que uno de los requisitos fundamentales resaltados en la literatura para su definición es que estas plataformas representan el rol de intermediarias.

Gráfico 5. Perfil del crowdfunding en España según modelos (2015 vs. 2018).

Fuente: elaboración propia

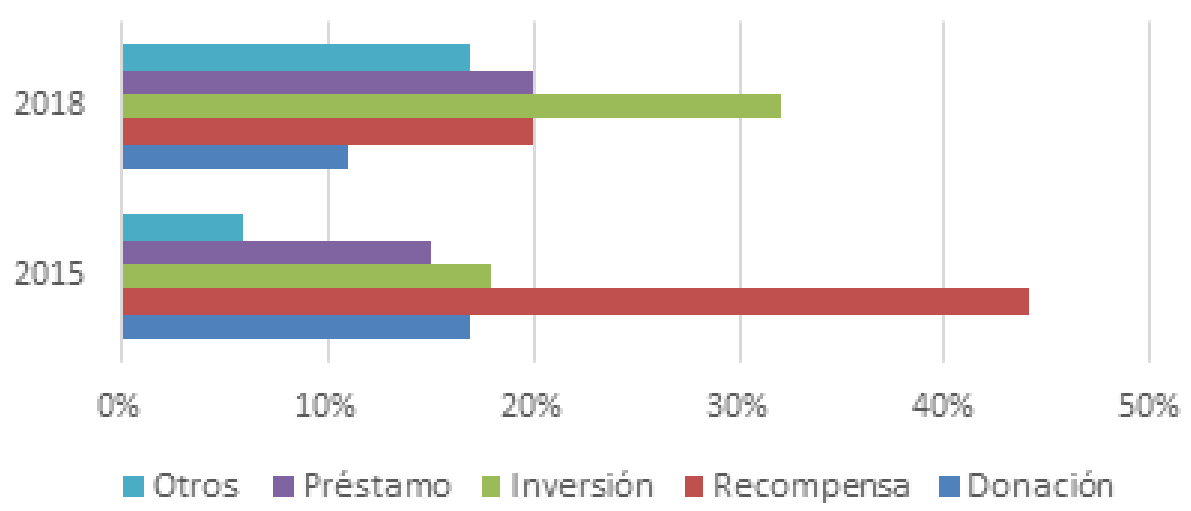

Es lícito preguntarse si el crowdfunding sigue siendo una forma de consumo colaborativo en la actualidad o si tan solo son colaborativas algunas de sus formas. $\mathrm{Si}$ nos referimos al fenómeno hace apenas cuatro años, las plataformas de recompensas suponían un 44\% del total, es decir, casi la mitad. El crowdfunding era un sector que podíamos identificar con ese modelo de "discos y películas" al que se sumaron muchas otras iniciativas de financiación alternativa. Un modelo que acogía bajo su paraguas a practicantes de deportes minoritarios, miembros de la "cultura friki" (Martínez, 2014), investigadores precarios, reporteros interesados en hacer periodismo libre alejado de fondos de inversión, o "emprendedores culturales", al mismo tiempo que compartían espacios digitales con personalidades famosas como Neil Young o instituciones prestigiosas como la NASA. La imagen en 2018, por el contrario, es la de un sector basado en la inversión y en las transacciones financieras (Gráfico 5). Como se aprecia en el siguiente gráfico el $42 \%$ de los proyectos alojados en las plataformas de inversión en España son de carácter inmobiliario (Gráfico 6). La entrada de las plataformas inmobiliarias ha supuesto un enorme impacto en el sector, modificándolo cualitativamente y generándonos preguntas acerca del carácter definitorio del mismo, así como desconfianzas y temores ante posibles casos de fraude (Hernández, 2017).

Gráfico 6. Proyectos alojados en las plataformas de inversión en España, 2018.

Fuente: elaboración propia

\begin{tabular}{|r|c|}
\hline \multirow{2}{*}{ Inmobiliario } & \\
\cline { 2 - 2 } Start-ups & $42 \%$ \\
\hline PYMES & $23 \%$ \\
\hline PYMES y Start-ups & $19 \%$ \\
\hline Emprendimiento & $10 \%$ \\
\hline Solidaridad & $3 \%$ \\
\hline
\end{tabular}


El trabajo desde las narrativas liberadoras o desde la autoexplotación

A lo largo de los años de irrupción y desarrollo del fenómeno del crowdfunding, encontramos numerosos blogs e informes corporativos que lo describen al tiempo que promocionan como un modelo de negocio exitoso capaz de recaudar ingentes cantidades de dinero, ofrecer alternativas a toda la sociedad o incluso de crear empleo. Universo Crowdfunding estima que en 2018 ha generado un total de 41.398 puestos de trabajo (Figura 2).

Figura 2. Estimación de puestos de trabajo creados por el crowdfunding en España, 2018.

Fuente: González y Ramos (2018, p. 12)

\begin{tabular}{|c|c|c|c|}
\hline Umbral $50.000 €$ & Umbral $20.000 €$ & Umbral 12.500€ & Umbral 9.000€ \\
\hline $\mathbf{3 . 1 9 4}$ & $\mathbf{7 . 9 8 5}$ & $\mathbf{1 2 . 7 7 6}$ & $\mathbf{1 7 . 4 4 3}$ \\
\hline Total recaudado/50.000€ & Total recaudado/20.000€ & Total recaudado/12.500€ & Total recaudado/9.000€ \\
\hline
\end{tabular}

Aunque los autores asumen las limitaciones de estas estimaciones y reconocen la imposibilidad de dar un dato real al respecto, se aventuran a afirmar que el crowdfunding genera una gran cantidad de puestos laborales "de calidad". De este modo, contribuyen al discurso extendido socialmente que considera al crowdfunding como un fenómeno positivo, sin casi defectos, que merece ser promovido económica y políticamente.

Siguiendo con la cuestión de los puestos de trabajo generados, los autores afirman:

En otros muchos casos el cálculo del umbral obedece más a prácticas de auto-empleo y tiene que ver con pre-ventas de libros, música o cualquier objeto tangible. En este caso no se está creando un puesto de trabajo en sí, sino que más bien se está financiando un empleo ya creado o se está favoreciendo una actividad económica específica (González y Ramos, 2018, p. 12).

La relación entre la calidad del empleo y lo aquí resaltado es especialmente contradictoria. Volviendo a los "emprendedores culturales", parece claro que lo que buscan es principalmente financiar los gastos derivados de actividades tales como la grabación de un disco. Aunque el crowdfunding permite a los promotores publicitarse, nada asegura que tras alcanzar ese objetivo de financiación tengan garantizada una gira de conciertos o poder vivir del proyecto. Esta perspectiva olvida cuestiones estructurales importantes, como quiénes están promoviendo proyectos, en este caso, los “emprendedores culturales", el escalafón más bajo de la precariedad en el mundo de la cultura y las artes. Pero también ignora análisis fundamentales para el ámbito del consumo colaborativo.

El "prosumidor colaborativo" (Alonso, 2017) ofrece y consume bienes y servicios por motivaciones más allá de lo económico. La idea de la remuneración ha ido cambiando desde sociedades previas a lo que Schiller llamó "capitalismo digital" hasta la actualidad (Frayssé y O’ Neil, 2015) (Véase en esta revista el monográfico dedicado al análisis del capitalismo digital, Jiménez y Rendueles, 2020). Los "prosumidores colaborativos" lanzan proyectos de crowdfunding envueltos en ideas relacionadas con "conectar con las personas" (Palmer,
2013), realizar proyectos independientes o formar parte de algo más grande. Algunos autores que se refieren a las dinámicas de prosumo en las economías colaborativas argumentan que éste no tiene coste para el prosumidor (Ritzer y Jurgenson, 2010). Esta es una idea debatible. Una campaña de crowdfunding dura una media de 40 días. La elaboración del proyecto implica necesariamente un tiempo dedicado a observar otros proyectos y conocer el medio en que se va a desarrollar, así como decidir en qué plataforma se va a lanzar. También planificarla, subir contenido a redes sociales diariamente, pensar en alternativas offline de recaudación, que implica contactar con medios de comunicación locales o llamadas a amigos y familiares. Además del propio diseño del proyecto que incluye la grabación de un vídeo de campaña entre otras cuestiones. Por otro lado, contestar a las preguntas lanzadas en los espacios interactivos de la plataforma o en las redes sociales. Todo esto es trabajo en sí en forma de prosumo y sin remuneración o al menos, podríamos decir que lo que existe es una esperanza de obtener remuneración, sabiendo que es posible no alcanzar el objetivo de financiación. El resultado es el "proletariado creativo" no pagado o mal pagado, sin ningún tipo de derechos o de estabilidad laboral (Arvidsson et al., 2008), que no solo realiza una gran cantidad de trabajo no considerado como tal sino que además debe estar pendiente de su dispositivo móvil en todo momento.

Cochoy (2015) plantea que el "prosumidor colaborativo" de crowdfunding a menudo lanza una campaña buscando obtener recursos suficientes para llevar a cabo su proyecto, pero al pasar los días se va dando cuenta de que el precio de lanzar una campaña es alto en términos de trabajo no pagado. Además, en el caso de que la campaña sea extremadamente exitosa aparecen otro tipo de problemas. Double Fine Adventure, el videojuego lanzado por Tim Schafer, ex colaborador de George Lucas en LucasArts, y financiado en Kickstarter, consiguió 3.336.371\$ cuando solo había pedido 400.000\$. En total, 87.142 personas financiaron el proyecto, que es el total de mecenas que solicitaron sus recompensas. Es decir, Tim Schafer debía entregar 87.142 recompensas, que incluían posters firmados, mini retratos personalizados con la cara del mecenas, cenas con los creadores 
del videojuego o el envío del producto a sus hogares. El proyecto fue lanzado en 2012 y esperaba entregar las recompensas en octubre del mismo año. Tardó cuatro años en terminar el trabajo (Schafer, 2012).

En definitiva, la no conciencia de estar realizando un trabajo no remunerado económicamente, sumado a otros ejes de explotación, tales como la imposibilidad de desconexión de los aparatos móviles, la importancia de mantener una buena reputación online y la propia exposición a perderla en espacios donde los promotores se vuelven vulnerables provoca que estemos hablando de formas de trabajo que dudosamente pueden considerarse de calidad. La pregunta pertinente al respecto es dónde ponemos la línea de la explotación (Ritzer y Jurgenson, 2010).

\section{Conclusiones}

España es uno de los lugares donde el crowdfunding se ha desarrollado de manera especialmente extensa. También es uno de los países pioneros en los que se implementó una regulación del crowdfunding (Ley 5/2015, de 27 de abril, de fomento de la financiación empresarial, 2015). No hay que olvidar que este no fue el único intento de regular las acciones mediadas por el crowdfunding. Tras la realización de las elecciones europeas, el Tribunal de Cuentas investigó la financiación de Podemos, que se había basado en el micromecenazgo desde su propia página web. El partido no había tenido en cuenta en la presentación de sus cuentas que los mecenas habían financiado su campaña de manera anónima en muchas ocasiones y, además, habían presentado el dinero obtenido desde una cuenta PayPal, cuando debían de haber recaudado ese dinero en la cuenta bancaria que el Tribunal de Cuentas tenía asociada a Podemos. Esto abrió un debate sobre la transparencia de las campañas políticas que utilizaban esta nueva herramienta de financiación, lo que trajo consigo la modificación de la Ley de Financiación de Partidos en el año 2015 (Ley Orgánica 8/2007, de 4 de julio, sobre financiación de los partidos políticos, 2007). España es por tanto pionera en la legislación de actividades relacionadas con el crowdfunding, algo que obedece sin lugar a dudas a la fuerte expansión de este fenómeno en nuestro país.

Es relevante peguntarnos sobre la influencia de los contextos socioeconómicos y su relación con la forma en que se constituye el crowdfunding en nuestros países. Parece lógico observar una conexión entre la crisis financiera global y las políticas de recortes en la conformación de las primeras formas de crowdfunding, vinculadas al modelo de recompensas. Al igual que el crecimiento acelerado de empresas inmobiliarias se da a la par que la existencia de la burbuja del alquiler que está viviendo España en la actualidad, también influida de hecho, por otras iniciativas de consumo colaborativo como Airbnb (Gil, 2017). Al igual que es importante preguntarnos por las nuevas formas de infraempleo generado por el crowdfunding o si existen diferencias entre aquel prosumo relacionado con el modelo de recompensas frente a otros conectados con las formas financieras del sector.
La realización de trabajos académicos meramente descriptivos del sector es especialmente relevante en el actual periodo histórico en el que los fenómenos digitales modifican nuestro comportamiento a la vez que evolucionan a un ritmo frenético, cambiando sus propios objetivos, formas y filosofías de fondo. La necesidad de estudiar de esta manera no solo el crowdfunding, sino otros fenómenos de lo colaborativo, se vuelve cada vez más acuciante si pensamos en la información que estamos obteniendo de ellos, basada en estudios corporativos que buscan limpiar la imagen de estos sectores, vendiéndolos como fenómenos puros en intención y comportamiento, así como prometedores desde una perspectiva económica. Plantear críticas se torna complejo cuando la casi totalidad de estudios ha nacido de las propias empresas y consultoras. Es por ello necesario publicar estudios científicos desde espacios académicos neutrales que puedan dar constancia real de las características del sector y que se alejen de estas narrativas de exaltación del éxito.

Por otro lado, las narrativas que exaltan el crowdfunding se centran en criterios económicos, sin embargo, es necesario dotar a este fenómeno de herramientas analíticas de carácter cualitativo que permitan relacionarlo con problemáticas sociales de actualidad. Una propuesta podría implicar alejarse del objetivo de investigación que suelen plantear los estudios corporativos y los nacidos del marketing, conocer qué plataformas recaudan más recursos financieros. Desde el punto de vista de las ciencias sociales es más relevante pensar en quiénes son nuestras plataformas, qué tipo de dinámicas prosumidoras se generan entre los mecenas y promotores o con qué perfiles sociales los relacionamos. La Comisión Europea está ejerciendo presión a los países de la eurozona para regular el sector del crowdfunding financiero (préstamo e inversión), dejando de lado a las plataformas de donación y recompensas que siguen manteniéndose fuera de las exigencias de un marco regulatorio, lo cual no implica que no deban o quieran ejercer su actividad bajo una legislación acorde a sus necesidades.

Parece, por la trayectoria del sector en nuestros países, que estuviéramos ante el desarrollo de dos fenómenos paralelos, diferentes, aunque similares, el crowdfunding financiero y el no financiero. El primero recoge el interés de instituciones gubernamentales, así como de la Comisión Europea, quienes han pretendido regular su actividad e incluso limitarla. Este modelo ha sembrado preocupación entre entidades bancarias como el Banco Bilbao Vizcaya Argentaria (BBVA) que ha resaltado en varios informes que es un sector disruptivo para la Banca (BBVA Research U.S. Unit, 2013; Cuesta et al., 2014). Prácticamente la totalidad de las plataformas financieras están promovida por empresas privadas, algo que contrasta con otros sectores como el de la donación, que contaba en 2015 con un $57 \%$ de entidades no lucrativas y con un $7 \%$ de entidades de carácter público. $\mathrm{O}$ con respecto al crowdfunding de recompensas, para el mismo año, un $8 \%$ de las plataformas eran ONGDs o fundaciones sin ánimo de lucro. El valor de estos dos modelos se basa en los flujos monetarios que son capaces de canalizar. Tal y como se ha explicado con 
anterioridad, lo financiero mueve mucho más capital económico y del mismo modo, encontramos un perfil de mecenas y de inversor muy diferenciado de los modelos no financieros. Todo ello nos acerca a preguntas sobre el carácter colaborativo de estas empresas, su pertenencia a las narrativas liberadoras, así como a los perfiles sociales que lanzan proyectos en cada una de estas plataformas. Aunque existen algunas iniciativas como Pentian, que permiten la inversión con retorno monetario para la financiación de proyectos editoriales, podemos observar una tendencia a través de la cual los emprendedores culturales y sectores sociales precarizados o afines lanzarán proyectos de recompensas frente a otros perfiles más relacionados con la innovación, que optarán por financiarse a través del préstamo o inversión. Una posible hipótesis para futuras investigaciones puede estar relacionada precisamente con esta cuestión, la segregación por clase social de los promotores de proyectos. Esto es que personas con más altas expectativas sociales, con una mayor red de contactos influyentes, procedentes de clases sociales más altas, sean quienes lanzan proyec- tos de inversión frente quienes centran su acción en el crowdfunding de recompensas.

Además, impacta observar como en tan solo cuatro años el perfil del crowdfunding ha cambiado tanto. Si bien en 2015 observábamos un sector asociado a las narrativas liberadoras afines a las economías colaborativas, en 2019 la cara del crowdfunding es la de las plataformas de carácter inmobiliario que, si bien pueden adoptar y adaptar los discursos de sus predecesoras, son también ampliamente cuestionadas. Sin duda, este es un aspecto al que prestar una especial atención. Para finalizar, la información es aún escasa y se necesitan muchos más esfuerzos por caracterizar este sector que es sin duda heterogéneo, que muestra diferencias claras frente a otras formas de economía colaborativa en las que predominan las empresas unicornio y que modifica su perfil de manera muy acelerada a medida que cambian los escenarios socioeconómicos de nuestro país. Se necesitan herramientas para describir el sector de manera fidedigna y a 10 años de su surgimiento estamos aún lejos de ellas.

\section{Referencias}

Alonso, L. E. (2017). Consumo colaborativo: Las razones de un debate. Revista Española de Sociología, 26(1), 87-95. https:// doi.org/10.22325/fes/res.2017.4

Arvidsson, A. Bauwens, M. y Peitersen, N. (2008). The crisis of value and the ethical economy. Journal of Futures Studies, 12(4), 9-20.

BBVA Research U.S. Unit. (2013). US economic outlook second quarter 2013. Bilbao: BBVA Research. Recuperado de: https:// www.bbvaresearch.com/wp-content/uploads/migrados/1306_EEUUOutlook_2Q13_tcm348-392269.pdf

Beck, U. y Kaldor, M. (2015). Conclusion: Towards a European Spring? En M. Kaldor y S. Selchow, y T. Murray-Leach, Subterranean Politics in Europe: A collaborative research project on Europe (pp. 231-238). Londres: Springer.

Belk, R. (2014). You are what you can access: sharing and collaborative consumption online. Journal of Business Research, 67, 1595-1600. https://doi.org/10.1016/j.jbusres.2013.10.001

Bollier, D. y Helfrich, S. (2015). Patterns of Commoning. Londres: The Commons Strategy Group and Off the Common Press.

Botsman, R. y Rogers, R. (2010). What's Mine is Yours: The Rise of Collaborative Consumption. Nueva York: HaperBusiness.

Cochoy, F. (2015). Consumers at Work, or Curiosity at Play? Revisiting the Prosumption/Value Cocreation Debate with Smartphones and two-dimensional bar codes. Marketing Theory, 15(2), 133-153. https://doi.org/10.1177/1470593114540676

Consumo Colaborativo (2017). Directorio de proyectos. [Mensaje en un blog]. Recuperado de: https://www.consumocolaborativo. com/directorio-de-proyectos/

Cuesta, C. Fernández de Lis, S. Roibas, I. Rubio, A. Ruesta, M. Tuesta, D. y Urbiola, P. (2014). Crowdfunding en $360^{\circ}$ : Alternativas de financiación en la era digital. Bilbao: BBVA Research. Recuperado de: https://www.bbvaresearch.com/wpcontent/uploads/2014/10/Observatorio-crowdfunding-vf.pdf

Denegri-Knott, J. and Zwick, D. (2012). Tracking prosumption on eBay: desire, enchantment, and the challenge of slow remcDonaldization. American Behavioral Scientist, 56(4), 439-458. https://doi.org/10.1177/0002764211429360

European Commission (2013). Crowdfunding innovative ventures in Europe. The financial ecosystem and regulatory landscape. Bruselas: Comisión Europea. Recuperado de: https:/ec.europa.eu/digital-single-market/news/crowdfunding-innovativeventures-europe-financial-ecosystem-and-regulatory-landscape-smart

Fernández-Savater, A. y Flesher, C. (2016). Life after the squares: Reflections on the consequences of the Occupy movements. Social Movement Studies, 16(1), 119-151. https://doi.org/10.1080/14742837.2016.1244478

Frayssé, O. y O’ Neil, M. (2015). Introduction: Hacked in the USA: Prosumption and digital labour. En O. Frayssé y M. O’Neil, Digital labour and prosumer capitalism: The US matrix (pp. 1-19). Londres: Palgrave Macmillan.

Gil, E. (2019). Is Crowdfunding (and Sharing Economy) a Type of Activism? En B. Tejerina, C. Miranda de Almeida, I. Perugorría, Sharing Society: The impact of collaborative collective actions in the transformation of contemporary societies. (pp. 172185). Bilbao: Universidad del País Vasco.

Gil, J. (2017). Desigualdades, límites y posibilidades para la transformación del capital en las economías colaborativas. Redes. Com, 15, 32-67. https://doi.10.15213/redes.n15.p33

González, Á. y Ramos, J. (2018). Financiación Participativa en España 2018. Madrid: Universo Crowdfunding y Universidad Complutense de Madrid y UDELAR- Montevideo. Recuperado de: https://www.universocrowdfunding.com/wp-content/ uploads/UC_Informe-anual-del-Crowdfunding-ES-EN-2018_XX92-FO2P-XZA1-32IK.pdf

Hernández, E. (2017). El crowdfunding inmobiliario mediante contratos de cuentas en participación: Una fórmula de inversión participativa ¿alegal o prohibida? Revista de estudios europeos, 70, 126-146.

Jiménez, A. y Rendueles, C. (2020) (coord.). Capitalismo Digital. Teknokultura. Revista de Cultura Digital y Movimientos Sociales, 17(2) https://doi.org/10.5209/tekn.70378

Lasén, A. (2006). Lo social como movilidad: usos y presencia del teléfono móvil. Política y Sociedad, 43(2), pp. 96-105 
Lee, A. (2 noviembre, 2013). Welcome to the Unicorn Club: Learning from Billion-Dollar Startups. [Mensaje en un blog]. Recuperado de: https://techcrunch.com/2013/11/02/welcome-to-the-unicorn-club/

Ley Orgánica 8/2007, de 4 de julio, sobre financiación de los partidos políticos, BOE (2007). Recuperado de: https://www.boe. es/boe/dias/2007/07/05/pdfs/A29010-29016.pdf

Ley 5/2015, de 27 de abril, de fomento de la financiación empresarial., BOE, 36600 (2015). Recuperado de: https://www.boe.es/ boe/dias/2015/04/28/pdfs/BOE-A-2015-4607.pdf

Ley 2012, de 5 de abril. Statutes at large, 5 de abril de 2012, núm. 126, pp. 126 a 306. Recuperado de: https://www.sec.gov/ spotlight/jobs-act.shtml

Manzini, E. (2019). Politics of the Everyday. Nueva York: Bloomsbury Publishing Plc.

Martínez, C. (2014). La Búsqueda de Nuevos Valores, Referentes y Modelos en un Mundo Líquido: El Refugio de la Cultura Friki en España (Tesis Doctoral, Universidad Pontificia de Salamanca). Recuperado de: http://summa.upsa.es/viewer.vm?id=0000 033145ypage=1 ysearch=ylang=esyview=main

O’Neil, M. y Frayssé, O. (2015). Digital Labour and Prosumer Capitalism: The US Matrix. Londres: Palgrave Macmillan.

Otto, C. (2017, 20 febrero). De sueldos millonarios a las «fintech»: Los banqueros que se han pasado a las «startups». El Confidencial. Recuperado de: https://www.elconfidencial.com/tecnologia/2017-02-20/banqueros-startups-angel-cano-bbvabancaja-indexa-capital-the-crowd-angel-fintech_1331317/

Palmer, A. (febrero, 2013). El arte de pedir. En TED Talks. [Vídeo online]. Recuperado de: https://www.ted.com/talks/amanda_ palmer_the_art_of_asking?language $=\mathrm{es}$

Rey, A. (2016, 20 mayo). Si una peseta diera cada español. El Mundo. Recuperado de: https://www.elmundo.es/blogs/elmundo/ personajazos/2016/05/20/si-una-peseta-diera-cada-espanol.html

Ritzer, G. y Jurgenson, N. (2010). Production, consumption, prosumption: the nature of capitalism in the age of the digital «prosumer». Journal of Consumer Culture, 10(1), 13-36. https://doi.org/10.1177/1469540509354673

Rowan, J. (2010). Emprendizajes en cultura. Discursos, instituciones y contradicciones de la empresarialidad cultural. Madrid: Traficantes de Sueños.

Sabadell, D. (2019, 23 julio). La mayor sentencia contra Deliveroo dicta que 532 riders eran falsos autónomos. El Salto Diario. Recuperado de: https://www.elsaltodiario.com/deliveroo/la-mayor-sentencia-deliveroo-dicta-532-riders-falsos-autonomos

Sastre, E. (2015). El crowdfunding de recompensa cultural en España. Análisis de los proyectos culturales publicados en Verkami desde el punto de vista de los autores (Trabajo de Fin de Máster, Universitat de Barcelona). Recuperado de: http://www. gestioncultural.org/ficheros/El_crowdfunding_de_recompensa_cultural_en_Espana.pdf

Schafer, T. (2012). Double Fine Adventure. [Campaña de Crowdfunding]. Recuperado de: https://www.kickstarter.com/projects/ doublefine/double-fine-adventure/description

Schor, J. (2014). Debating the Sharing Economy. Cambridge: A Great Transition Initiative. Recuperado de: https://greattransition. org/publication/debating-the-sharing-economy

Wardrop, R. Zhang, B. Raghavendra, R. y Gray, M. (2015). Moving mainstream. The European alternative finance benchmarking report. Cambridge: University of Cambridge y Ernst y Young. Recuperado de: https://www.jbs.cam.ac.uk/faculty-research/ centres/alternative-finance/publications/moving-mainstream/

Zafra, R. (2017). El entusiasmo. Precariedad y trabajo en la era digital. Barcelona: Anagrama. 